\title{
Appendix
}

The proof of Theorem proceeds by a series of lemmas.

Lemma 1 If the random indicator $A_{i}$ that is conditional on $X_{i}=x_{i}$ is Bernoulli $\left(1-\pi_{0}\left(x_{i}\right)\right)$, then $E\left(\Psi\left(z_{i} ; x_{i}\right) \mid X_{i}=x_{i}\right)=\pi_{0}\left(x_{i}\right)$.

Proof 1 We have

$$
E\left(A_{i} \mid Z_{i}=z_{i}, X_{i}=x_{i}\right)=P\left(A_{i}=1 \mid Z_{i}=z_{i}, X_{i}=x_{i}\right)=1-\Psi\left(z_{i} ; x_{i}\right) .
$$

By taking the expectation with respect to the density of $Z_{i}$ that is conditional on $X_{i}=x_{i}$, we obtain

$$
\begin{array}{r}
E\left(E\left(A_{i} \mid Z_{i}=z_{i}, X_{i}=x_{i}\right) \mid X_{i}=x_{i}\right)=E\left(1-\Psi\left(z_{i} ; x_{i}\right) \mid X_{i}=x_{i}\right), \\
E\left(A_{i} \mid X_{i}=x_{i}\right)=1-E\left(\Psi\left(z_{i} ; x_{i}\right) \mid X_{i}=x_{i}\right),
\end{array}
$$

and the result follows.

Lemma 2 For $x_{i} \in \mathcal{R}_{1}\left(x_{0}, \Delta_{0}\right)$, the bootstrap estimator $\widehat{\mu}\left(\Delta_{0}, B\right)$ is a weakly consistent estimator of $\pi_{01}$. That is,

$$
\lim _{N \rightarrow \infty} \lim _{B \rightarrow \infty} P\left(\left|\widehat{\mu}\left(\Delta_{0}, B\right)-\pi_{01}\right|>\epsilon \mid X_{i}=x_{i}\right)=0 \text { for any } \epsilon>0 .
$$

Proof 2 By Markov's inequality, it holds for any $\epsilon>0$ that

$$
\begin{aligned}
P\left(\left|\widehat{\mu}\left(\Delta_{0}, B\right)-\pi_{01}\right|>\epsilon \mid X_{i}=x_{i}\right) & \leq \frac{E\left[\left|\widehat{\mu}\left(\Delta_{0}, B\right)-\pi_{01}\right| \mid X_{i}=x_{i}\right]}{\epsilon} \\
& \leq \frac{E\left[\left|\widehat{\mu}\left(\Delta_{0}, B\right)-\mu_{\Delta_{0}}\left(x_{i}\right)\right| \mid X_{i}=x_{i}\right]}{\epsilon} \\
& +\frac{E\left[\left|\mu_{\Delta_{0}}\left(x_{i}\right)-\pi_{01}\right| \mid X_{i}=x_{i}\right]}{\epsilon} .
\end{aligned}
$$

$\widehat{\mu}\left(\Delta_{0}, B\right)$ is an unbiased estimator of $\mu_{\Delta_{0}}\left(x_{i}\right)$, and has zero variance as $B$ becomes large. That is,

$$
\lim _{B \rightarrow \infty} E\left[\left(\widehat{\mu}\left(\Delta_{0}, B\right)-\mu_{\Delta_{0}}\left(x_{i}\right)\right)^{2} \mid X_{i}=x_{i}\right]=\lim _{B \rightarrow \infty} \frac{\sigma_{\Delta_{0}}^{2}\left(x_{i}\right)}{B}=0 .
$$

Hence,

$\lim _{B \rightarrow \infty} E\left[\left|\widehat{\mu}\left(\Delta_{0}, B\right)-\mu_{\Delta_{0}}\left(x_{i}\right)\right| \mid X_{i}=x_{i}\right] \leq \lim _{B \rightarrow \infty} E\left[\left(\widehat{\mu}\left(\Delta_{0}, B\right)-\mu_{\Delta_{0}}\left(x_{i}\right)\right)^{2} \mid X_{i}=x_{i}\right]^{\frac{1}{2}}=0$.

On the other hand, when $x_{i} \in \mathcal{R}_{1}\left(x_{0}, \Delta_{0}\right)$ the expected dimension of the reference class $\boldsymbol{z}_{i}^{\Delta_{0}}$ as $N$ becomes large is $\lim _{N \rightarrow \infty} d_{i}^{\Delta_{0}}=\infty$. By applying the consistency assumption of $\widehat{\Psi}_{i}$ on the reference class $\boldsymbol{z}_{i}^{\Delta_{0}}$, we have that

$$
\lim _{N \rightarrow \infty} P\left(\left|\widehat{\Psi}_{i}\left(\boldsymbol{z}_{i}^{\Delta_{0}}\right)-\Psi\left(z_{i} ; x_{i}\right)\right|>\epsilon \mid X_{i}=x_{i}\right)=0 .
$$

Because $\left|\widehat{\Psi}_{i}\left(\boldsymbol{z}_{i}^{\Delta_{0}}\right)-\Psi\left(z_{i} ; x_{i}\right)\right| \leq 1$, the dominated convergence Theorem implies that

$$
\lim _{N \rightarrow \infty} E\left[\widehat{\Psi}_{i}\left(\boldsymbol{z}_{i}^{\Delta_{0}}\right)-\Psi\left(z_{i} ; x_{i}\right) \mid X_{i}=x_{i}\right]=0 .
$$


For $x_{i} \in \mathcal{R}_{1}\left(x_{0}, \Delta_{0}\right), E\left(\Psi\left(z_{i} ; x_{i}\right) \mid X_{i}=x_{i}\right)=\pi_{01}$ and

$$
\lim _{N \rightarrow \infty} \frac{E\left[\left|\mu_{\Delta_{0}}\left(x_{i}\right)-\pi_{01}\right| \mid X_{i}=x_{i}\right]}{\epsilon}=0 .
$$

Lemma 3 If $x_{i} \in \mathcal{R}_{1}\left(x_{0}, \Delta_{0}\right)$, then the bootstrap estimator $\widehat{\mathcal{B}}\left(\Delta, \Delta_{0}, B\right)$ is a weakly consistent estimator of the prediction bias $\mathcal{B}_{\Delta}\left(x_{i}\right)$. That is,

$$
\lim _{N \rightarrow \infty} \lim _{B \rightarrow \infty} P\left(\left|\widehat{\mathcal{B}}\left(\Delta, \Delta_{0}, B\right)-\mathcal{B}_{\Delta}\left(x_{i}\right)\right|>\epsilon \mid X_{i}=x_{i}\right)=0 \text { for any } \epsilon>0 \text {. }
$$

Proof 3 By Markov's inequality, we have for any $\epsilon>0$ that

$$
\begin{aligned}
P\left(\left|\widehat{\mathcal{B}}\left(\Delta, \Delta_{0}, B\right)-\mathcal{B}_{\Delta}\left(x_{i}\right)\right|>\epsilon \mid X_{i}=x_{i}\right) & \leq \frac{E\left[\left|\widehat{\mathcal{B}}\left(\Delta, \Delta_{0}, B\right)-\mathcal{B}_{\Delta}\left(x_{i}\right)\right| \mid X_{i}=x_{i}\right]}{\epsilon} \\
& \leq \frac{E\left[\left|\widehat{\mu}(\Delta, B)-\mu_{\Delta}\left(x_{i}\right)\right| \mid X_{i}=x_{i}\right]}{\epsilon} \\
& +\frac{E\left[\left|\widehat{\mu}\left(\Delta_{0}, B\right)-\pi_{01}\right| \mid X_{i}=x_{i}\right]}{\epsilon} .
\end{aligned}
$$

Because $\widehat{\mu}(\Delta, B)$ is an unbiased estimator of $\mu_{\Delta}\left(x_{i}\right)$ whose variance is asymptotically zero, the result follows from Lemma 2 and the fact that

$$
\lim _{B \rightarrow \infty} E\left[\left|\widehat{\mu}(\Delta, B)-\mu_{\Delta}\left(x_{i}\right)\right| \mid X_{i}=x_{i}\right] \leq \lim _{B \rightarrow \infty} E\left[\left(\widehat{\mu}(\Delta, B)-\mu_{\Delta}\left(x_{i}\right)\right)^{2} \mid X_{i}=x_{i}\right]^{\frac{1}{2}}=0 .
$$

Lemma 4 For $x_{i} \in \mathcal{R}_{1}\left(x_{0}, \Delta_{0}\right)$, the bootstrap estimator $\widehat{\Delta}_{0 i}^{\star}$ is a weakly consistent estimator of $\Delta_{0 i}^{\star}$. That is,

$$
\lim _{N \rightarrow \infty} \lim _{B \rightarrow \infty} P\left(\left|\widehat{\Delta}_{0 i}^{\star}-\Delta_{0 i}^{\star}\right|>\epsilon \mid X_{i}=x_{i}\right)=0 \text { for any } \epsilon>0 .
$$

Proof 4 The bootstrap sample variance is a weakly consistent estimator of the variance of $\widehat{\Psi}_{i}\left(\boldsymbol{z}_{i}^{\Delta}\right)$ and it follows from Lemma 3, that

$$
\lim _{N \rightarrow \infty} \lim _{B \rightarrow \infty} P\left(\left|\widehat{\operatorname{err}}\left(\Delta, \Delta_{0}, B\right)-\operatorname{err}\left(\widehat{\Psi}\left(\boldsymbol{z}_{i}^{\Delta}\right) \mid X_{i}=x_{i}\right)\right|>\epsilon \mid X_{i}=x_{i}\right)=0 .
$$

Therefore, the result follows from the continuous mapping Theorem and the fact that $\lim _{N \rightarrow \infty} \lim _{B \rightarrow \infty} P\left(\left|\arg \inf _{\Delta \geq \Delta_{0}} \widehat{\operatorname{err}}\left(\Delta, \Delta_{0}, B\right)-\arg \inf _{\Delta \geq \Delta_{0}} \operatorname{err}\left(\widehat{\Psi}\left(\boldsymbol{z}_{i}^{\Delta}\right) \mid X_{i}=x_{i}\right)\right|>\epsilon \mid X_{i}=x_{i}\right)=0$. 


\section{Proof of Theorem:}

Proof 5 We know that

$$
\begin{gathered}
\operatorname{MSE}\left(\widehat{\Psi}_{i}\left(\boldsymbol{z}_{i}^{\widehat{\Delta}_{0 i}^{\star}}\right) \mid \mathcal{R}_{1}\left(x_{0}, \Delta_{0}\right)\right)=\int_{\mathcal{R}_{1}\left(x_{0}, \Delta_{0}\right)} \operatorname{MSE}\left(\widehat{\Psi}_{i}\left(\boldsymbol{z}_{i}^{\widehat{\Delta}_{0 i}^{\star}}\right) \mid X_{i}=x_{i}\right) d P_{x_{i}}, \\
\operatorname{MSE}\left(\widehat{\Psi}_{i}(\boldsymbol{z}) \mid \mathcal{R}_{1}\left(x_{0}, \Delta_{0}\right)\right)=\int_{\mathcal{R}_{1}\left(x_{0}, \Delta_{0}\right)} \operatorname{MSE}\left(\widehat{\Psi}_{i}(\boldsymbol{z}) \mid X_{i}=x_{i}\right) d P_{x_{i}} .
\end{gathered}
$$

It suffices to show that

$$
\begin{aligned}
& \lim _{N \rightarrow \infty} \lim _{B \rightarrow \infty}\left[\operatorname{MSE}\left(\widehat{\Psi}_{i}\left(\boldsymbol{z}_{i}^{\widehat{\Delta}_{0 i}^{\star}}\right) \mid X_{i}=x_{i}\right)-\operatorname{MSE}\left(\widehat{\Psi}_{i}(\boldsymbol{z}) \mid X_{i}=x_{i}\right)\right] \leq 0 . \\
& \operatorname{MSE}\left(\widehat{\Psi}_{i}\left(\boldsymbol{z}_{i}^{\widehat{\Delta}_{0 i}^{\star}}\right) \mid X_{i}=x_{i}\right)-\operatorname{MSE}\left(\widehat{\Psi}_{i}(\boldsymbol{z}) \mid X_{i}=x_{i}\right)=\operatorname{err}\left(\widehat{\Psi}_{i}\left(\boldsymbol{z}_{i}^{\widehat{\Delta}_{0 i}^{\star}}\right) \mid X_{i}=x_{i}\right) \\
& -\operatorname{err}\left(\widehat{\Psi}_{i}\left(\boldsymbol{z}_{i}^{\Delta_{0 i}^{\star}}\right) \mid X_{i}=x_{i}\right) \\
& +\operatorname{err}\left(\widehat{\Psi}_{i}\left(\boldsymbol{z}_{i}^{\Delta_{0 i}^{\star}}\right) \mid X_{i}=x_{i}\right) \\
& -\operatorname{err}\left(\widehat{\Psi}_{i}(\boldsymbol{z}) \mid X_{i}=x_{i}\right) \text {. }
\end{aligned}
$$

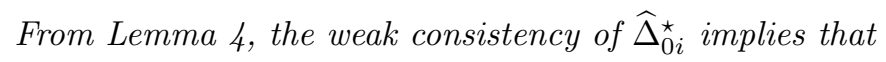

$$
\lim _{N \rightarrow \infty} \lim _{B \rightarrow \infty} \operatorname{err}\left(\widehat{\Psi}_{i}\left(\boldsymbol{z}_{i}^{\widehat{\Delta}_{0 i}^{\star}}\right) \mid X_{i}=x_{i}\right)-\operatorname{err}\left(\widehat{\Psi}_{i}\left(\boldsymbol{z}_{i}^{\Delta_{0 i}^{\star}}\right) \mid X_{i}=x_{i}\right)=0 .
$$

On the other hand, because $\Delta_{0 i}^{\star}$ is optimal tuning parameter, it follows that

$\operatorname{err}\left(\widehat{\Psi}_{i}\left(\boldsymbol{z}_{i}^{\Delta_{0 i}^{\star}}\right) \mid X_{i}=x_{i}\right)-\operatorname{err}\left(\widehat{\Psi}_{i}(\boldsymbol{z}) \mid X_{i}=x_{i}\right) \leq \operatorname{err}\left(\widehat{\Psi}_{i}\left(\boldsymbol{z}_{i}^{\Delta}\right) \mid X_{i}=x_{i}\right)-\operatorname{err}\left(\widehat{\Psi}_{i}(\boldsymbol{z}) \mid X_{i}=x_{i}\right)$

for any $\Delta \in\left[\Delta_{0}, \infty\right)$, which indicates that

$$
\begin{aligned}
\lim _{N \rightarrow \infty} \lim _{B \rightarrow \infty}\left[M S E\left(\widehat{\Psi}_{i}\left(\boldsymbol{z}_{i}^{\widehat{\Delta}_{i i}^{\star}}\right) \mid X_{i}=x_{i}\right)-\operatorname{MSE}\left(\widehat{\Psi}_{i}(\boldsymbol{z}) \mid X_{i}=x_{i}\right)\right] \\
\quad \leq \lim _{N \rightarrow \infty} \lim _{B \rightarrow \infty}\left[\operatorname{err}\left(\widehat{\Psi}_{i}\left(\boldsymbol{z}_{i}^{\Delta}\right) \mid X_{i}=x_{i}\right)-\operatorname{err}\left(\widehat{\Psi}_{i}(\boldsymbol{z}) \mid X_{i}=x_{i}\right)\right] \\
\quad=\lim _{N \rightarrow \infty} \lim _{B \rightarrow \infty}\left[M S E\left(\widehat{\Psi}_{i}\left(\boldsymbol{z}_{i}^{\Delta}\right) \mid X_{i}=x_{i}\right)-M S E\left(\widehat{\Psi}_{i}(\boldsymbol{z}) \mid X_{i}=x_{i}\right)\right]=0 .
\end{aligned}
$$

The facts that both $\lim _{N \rightarrow \infty} \operatorname{MSE}\left(\widehat{\Psi}_{i}(\boldsymbol{z}) \mid X_{i}=x_{i}\right)=0$ and $\lim _{N \rightarrow \infty} \operatorname{MSE}\left(\widehat{\Psi}_{i}\left(\boldsymbol{z}_{i}^{\Delta}\right) \mid X_{i}=x_{i}\right)=0$ follow from the consistency and the dominated convergence Theorem. 